\title{
Antimicrobial starch-citrate hydrogel for potential applications as drug delivery carriers
}

\author{
Suk Fun Chin ${ }^{1, *}$, Ain Nadirah Binti Romainor ${ }^{2}$, Suh Cem Pang ${ }^{1}$, Samuel Lihan ${ }^{1}$ \\ ${ }^{1}$ Faculty of Resource Science and Technology, Universiti Malaysia Sarawak, 94300, Kota Samarahan, Sarawak, Malaysia \\ ${ }^{2}$ School of Foundation Studies (PUScFS), Perdana University, MAEPS Building, MARDI Complex Jalan MAEPS Perdana, 43400, Seri Kembangan, Selangor, Malaysia
}

\section{A R T I C L E I N F O}

\section{Keywords:}

Starch-citrate hydrogel

Antimicrobial

Multiple antibiotic resistant (M.A.R)

Controlled release

\begin{abstract}
A B S T R A C T
Antimicrobial starch-citrate hydrogels were prepared by physical crosslinking of starch citrate, Poly(vinyl alcohol (PVA) and Polyethylene glycol (PEG) via the freeze-thaw technique. This starch-citrate hydrogel exhibited excellent antimicrobial activity against various bacteria strains such as Escherichia coli (gram negative), Staphylococcus pyogenes (gram negative), Salmonella thypimurium (gram positive) and Streptococcus aureus (gram positive). Most notably it showed antimicrobial activity against the multiple antibiotic resistant (M.A.R) superbugs (Klebsiella pneumoniae). Penicillin G loaded starch-citrate hydrogel was observed to exhibit a sustain release profile over 7 days. Penicillin G loaded starch-citrate hydrogel also exhibited synergistic antimicrobial activities against Salmonella thypimurium, Escherichia coli (E-coli) (O15:H7) and E-coli control (ATC 2925). The potential application of starch-citrate hydrogel as both antimicrobial agent and drug delivery carriers is therefore envisaged.
\end{abstract}

\section{Introduction}

Misuse and overuse of conventional antibiotics have led to increasing antibiotic resistance to the existing antibiotics and rapid emergence of new infections. Most conventional antibiotics (such as ciprofloxacin, penicillin and ceftazidime) do not physically damage the bacteria cell wall, therefore preserving the bacterial morphology and, as a consequence, the bacteria can easily develop resistance towards the antibiotics [1]. Recently, antimicrobial hydrogel has emerged as a new class of fascinating macromolecular antimicrobial agent, which is effective in preventing and combating multidrug-resistant infections [2]. Hydrogel is made up of three-dimensional (3D) networks which can hold large amount of water and swell without dissolution. Such properties afford the hydrogel to remain in place under physiological conditions while maintaining antimicrobial potency. Antimicrobial hydrogels work by causing damaging or disrupting the bacteria membranes. This physical damage on the bacteria cell walls prevents the microbes from developing resistance [3].

Currently, most of the antimicrobial hydrogels are either chitosanbased [4] or peptide-based [5]. However, the use of chitosan-based hydrogel could give rise to immunogenicity. On the other hand, peptide-based hydrogel has achieved very limited success in clinical trials, mainly due to their cytotoxicity, short half-live in vivo and high production cost. Various cationic synthetic polymers such as derivatives of poly(methyl methacrylate) [6], polyethylenimine [7] and polycarbonate [8] that mimic the amphiphilic structure and antimicrobial functionalities of peptides have been synthesized because their synthesis are simpler and easier to scale up as compared with peptides. However, most of these antimicrobial polymers reported in the literature are non-biodegradable, which have hindered their in vivo applications.

In this study, we have prepared starch-citrate hydrogel as the potential antimicrobial agent cum drug delivery carriers. Native sago starch was selected as a precursor material for hydrogel preparation as it is abundantly available, renewable, low cost and nontoxic in nature. Citric acid was functionalized onto the starch to form starch-citrate. Citric acid possess a tricarboxylic acid functional group which can pass through the bacteria cell membranes and act as a natural antimicrobial agent to suppress bacteria growth by lowering the intracellular $\mathrm{pH}$ of the microorganism cells [9]. Starch-citrate hydrogel demonstrated great antimicrobial activity against the multiple antibiotic resistant (M.A.R) superbugs (Klebsiella pneumoniae) demonstrating that it holds great potential to serve as an alternative antimicrobial agent for combating the drug-resistant infection. The synergistic antimicrobial effects of penicillin G loaded starch-citrate hydrogel against Salmonella thypimurium, Escherichia coli (E-coli) (O15:H7) and E-coli control (ATC 2925) could have a profound implication in reducing the amount of antibiotics use for infection treatments.

\footnotetext{
* Corresponding author.

E-mail address: sfchin@unimas.my (S.F. Chin).
} 